\title{
Electroformation of Giant Unilamellar Vesicles on Stainless Steel Electrodes
}

\author{
Valerio Pereno, ${ }^{\dagger}$ Dario Carugo, ${ }^{\dagger \dagger}$ Luca Bau, ${ }^{\dagger}$ Erdinc Sezgin,${ }^{\S}$ Jorge Bernardino de la Serna, ${ }^{\oplus}$ \\ Christian Eggeling, ${ }^{\S}$ and Eleanor Stride*,॰ \\ ${ }^{\dagger}$ Institute of Biomedical Engineering, Department of Engineering Science, University of Oxford, Oxford OX3 7DQ, U.K. \\ ${ }^{\ddagger}$ Mechatronics and Bioengineering Science Research Groups, Faculty of Engineering and the Environment, University of \\ Southampton, SO17 1BJ Southampton, U.K. \\ ${ }^{\S}$ Weatherall Institute of Molecular Medicine, MRC Human Immunology Unit, University of Oxford, Oxford OX3 9DS, U.K. \\ "Rutherford Appleton Laboratory, Central Laser Facility, Science and Technology Facilities Council, Research Complex at Harwell, \\ Harwell-Oxford, Didcot OX11 OFA, U.K.
}

\section{Supporting Information}

ABSTRACT: Giant unilamellar vesicles (GUVs) are well-established model systems for studying membrane structure and dynamics. Electroformation, also referred to as electroswelling, is one of the most prevalent methods for producing GUVs, as it enables modulation of the lipid hydration process to form relatively monodisperse, defect-free vesicles. Currently, however, it is expensive and time-consuming compared with other methods. In this study, we demonstrate that 1,2-dioleoyl-sn-glycero-3-phosphatidylcholine GUVs can be prepared readily at a fraction of the cost on stainless steel electrodes, such as commercially available syringe needles, without any evidence of lipid oxidation or hydrolysis.

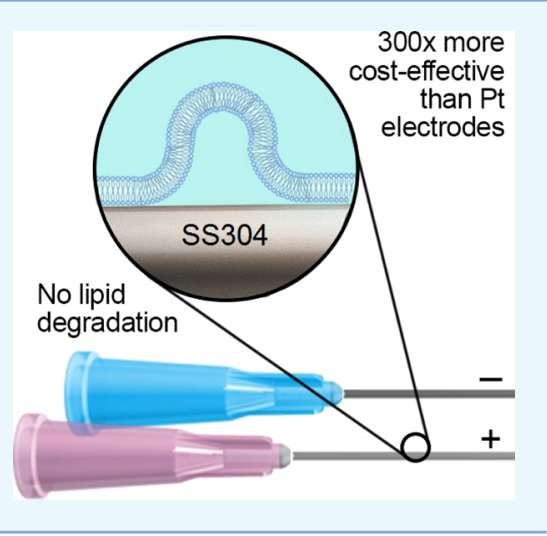

\section{INTRODUCTION}

Giant unilamellar vesicles (GUVs), often referred to as giant liposomes, are fluid-filled membranes that offer a useful basic model of a biological cell. GUVs have been widely used as models to study the fluid-fluid and gel-fluid phase coexistence of membrane lipids, ${ }^{1-6}$ membrane transport phenomena, ${ }^{7-12}$ the behavior of native membranes, ${ }^{13-16}$ the structure of early cells and protocells, ${ }^{17}$ and more recently, cells' biological activity. ${ }^{10}$ In its simplest form, a GUV consists of a single amphiphilic lipid bilayer that encloses an aqueous solution.

Among the multitude of production methods developed over several decades, ${ }^{18-20}$ lipid-film hydration, ${ }^{21}$ electroformation, ${ }^{22}$ lipid emulsification, ${ }^{23,24}$ and microfluidic-based methods, such as fluid jetting ${ }^{25}$ and hydrodynamic flow focusing, ${ }^{26}$ are the most widely used. While the literature on vesicle formation is rich with established formation protocols, each technique has its own inherent drawbacks and trade-offs according to the application. The factors affecting the choice of a specific protocol are technical expertise, the need for specialist equipment, and the degree to which vesicle characteristics need to be tuned. Of the methods mentioned above, electroformation is one of the most widespread. ${ }^{18}$

Electroformation of GUVs was pioneered by Angelova and Dimitrov in $1986 .{ }^{22}$ It involves modulating the spontaneous swelling of lipids within an aqueous solution using an externally applied electric field (Figure 1). Typically, a solution of lipids dissolved in an organic solvent is deposited on two electrodes of indium tin oxide (ITO)-coated glass or platinum. Following solvent evaporation, the electrodes are placed in contact with an aqueous solution. Subsequently, an alternating potential difference is applied across the electrodes, stimulating the swelling process of the hydrated lipid layer. The main advantages of electroformation are that it requires comparatively little technical expertise to implement and that it yields spherical, relatively monodisperse and unilamellar lipid vesicles. Set against this, however, is the cost of the electrodes, which limits the scalability of the technique.

In this article, we present and validate a simple electrode modification in the standard electroformation protocol that reduces the cost and time of chamber preparation while improving scalability. 1,2-Dioleoyl-sn-glycero-3-phosphatidylcholine (DOPC) was chosen as a representative unsaturated phospholipid, which is more prone to oxidation than saturated phospholipids, such as 1,2-distearoyl-sn-glycero-3-phosphocholine (DSPC), 1,2-dipalmitoyl-sn-glycero-3-phosphocholine (DPPC), and 1,2-dimyristoyl-sn-glycero-3-phosphocholine (DMPC), to prove that stainless steel does not affect its structural integrity.

Received: November 14, 2016

Accepted: March 3, 2017

Published: March 16, 2017 


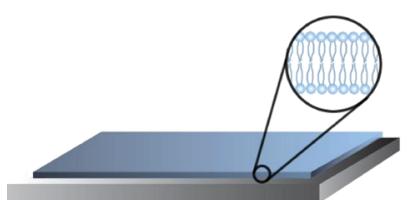

dry lipid film

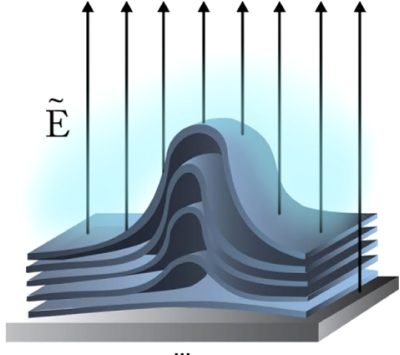

swelling

Figure 1. Schematic of vesicle electroformation from a dry lipid bilayer deposited on a substrate. Vesicles are formed upon hydration and the application of an alternating electric field, $\tilde{E}$ (not to scale).

Table 1. Comparison of Costs, Advantages, and Disadvantages of Using Stainless Steel Electrodes and Platinum Electrodes during Electroformation ${ }^{a}$

\begin{tabular}{|c|c|c|c|c|c|c|}
\hline & $\operatorname{cost}^{b}$ & disposable & scalable & machinable & cleaning required & consistent electrode separation \\
\hline platinum & $\sim £ 584$ & expensive & expensive & yes & yes & difficult \\
\hline stainless steel & $\sim £ 2.00$ & yes & yes & yes & only if reused & yes \\
\hline ITO-coated glass & $\sim £ 27.00$ & yes & yes & no & yes & yes \\
\hline
\end{tabular}

${ }^{a}$ A detailed cost breakdown of the electroformation chamber is provided in the Supporting Information. ${ }^{b}$ Calculated for five pairs of $40 \mathrm{~mm}$ cylindrical electrodes with a diameter of $0.8 \mathrm{~mm}$ or an ITO surface of equivalent area $\left(10 \mathrm{~cm}^{2}\right)$.

(a)

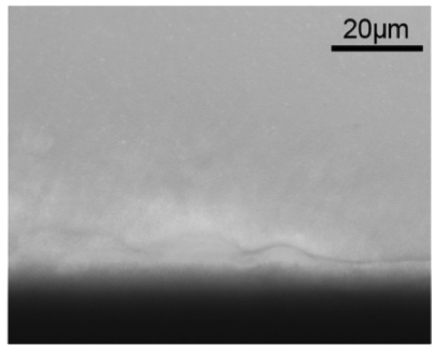

(b)

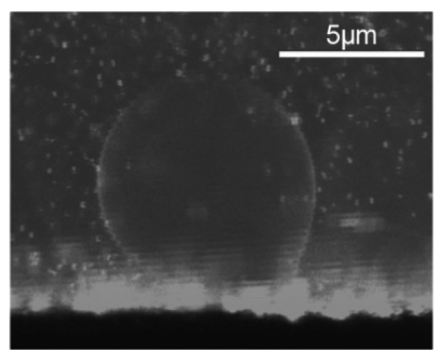

(c)

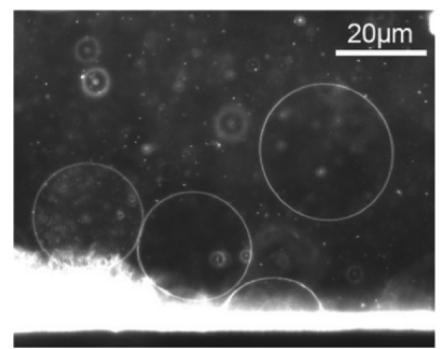

Figure 2. Vesicles swelling from electroformation electrodes: (a) hydrated lipid layer commencing the swelling process, (b) DiI-labeled single-vesicle swelling while attached to the electroformation electrodes, and (c) vesicles forming, swelling, and detaching along the electrode.

Stainless steel electrodes, such as injection needles, provide a significantly lower-cost alternative to platinum and ITO electrodes, which are the established electrode materials reported in the electroformation literature. Other studies have also reported electroformation of vesicles on interdigitated and nonconductive substrates, ${ }^{27}$ albeit with limited adoption rates.

Stainless steel electrodes provide numerous advantages. First, the rigidity of stainless steel compared to that of platinum reduces the risk of bending, improving control over the electrode-separation distance, thus leading to a more uniform electric field. In addition, disposable needles do not require cleaning in an ultrasound bath, ${ }^{28}$ are readily available, and do not require the modification of current chambers.

The wide cost disparity between stainless steel and platinum enables two secondary modifications to the electroformation protocol: the use of longer electrodes and chamber parallelization. ${ }^{29}$ These lead, in turn, to an increase in the overall vesicle production, concurrent electroformation, and multiparametric testing at high throughput.

While a direct comparison between electroformation methods is complex, Table 1 provides an overview of the merits and drawbacks of different electrode materials used in the electroformation of vesicles. To populate the table, the electrode surface area was fixed across all methods. The cost comparison was carried out on the face value of the electrodes, without taking into account the cost of the purpose-built polyoxymethylene electroformation chamber. A full cost breakdown of the electroformation chamber is provided in Table S1. It is noteworthy that, if the stainless steel electrodes were disposed of after every electroformation experiment, it would take approximately 292 experiments to match the cost of the platinum electrodes-ignoring overheads, such as the time and cost of cleaning.

In the experiments reported here, these features are exploited using a purpose-built electroformation device, which consists of a polyoxymethylene base containing five electroformation chambers and a lid to hold the electrodes in position.

To validate the proposed technique, vesicles were characterized in terms of their size distribution, lipid packing and unilamellarity of their membranes. In addition, the constituents of the aqueous electroformation solution were analyzed using inductively coupled plasma optical emission spectroscopy (ICP-OES) to test whether any ions are released from the electrodes during electroformation. Lipid degradation was assessed by NMR.

\section{RESULTS AND DISCUSSION}

GUV Production and Imaging. Electroformation visualization chambers have been used to monitor and verify the effective production of vesicles. ${ }^{30}$ To assess the formation of vesicles on stainless steel electrodes, swelling was visualized in situ (Figure 2a) using a second specially designed electroformation chamber with optical access using the protocol 
(a)

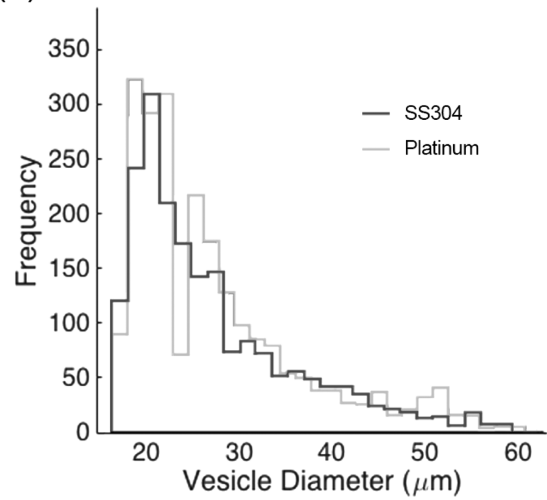

(b)

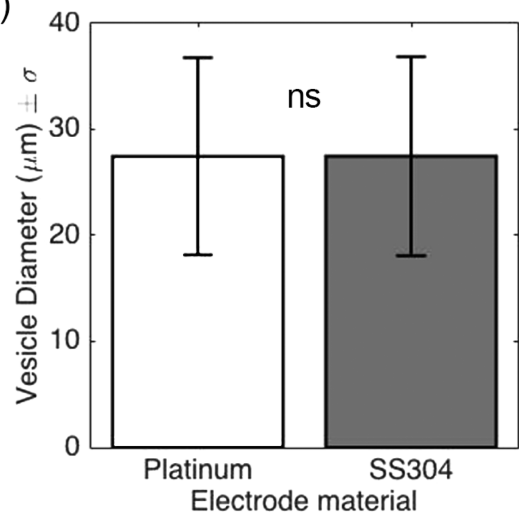

Figure 3. (a) Size distribution of DOPC vesicles as a function of electrode material. The white bars represent vesicles formed using platinum wires as electrodes, whereas the gray bars represent vesicles formed using stainless steel electrodes. (b) Comparison of average vesicle diameters ( \pm standard deviation) of platinum and stainless steel electrodes. Three independent experiments were conducted, and over 1500 vesicles were analyzed for each electrode material.

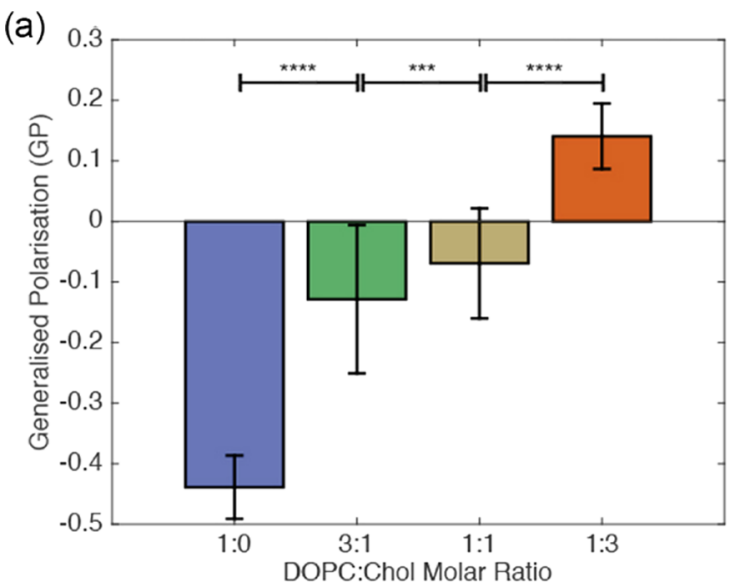

(b)

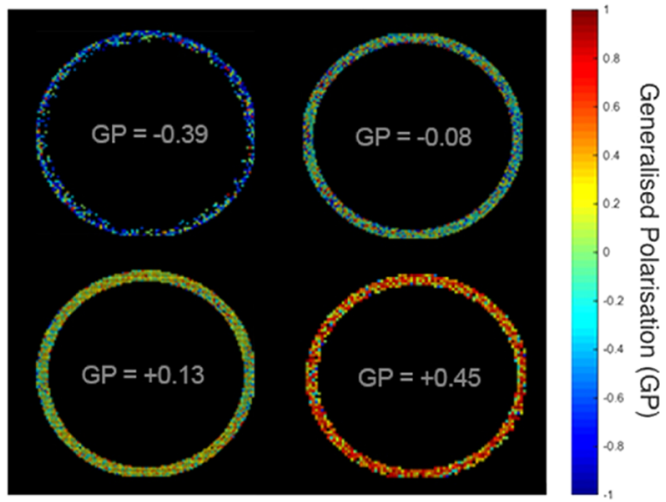

Figure 4. Simultaneous electroformation of vesicles with dissimilar membrane properties. (a) Generalized polarization (GP) of the vesicles as a function of initial lipid-film formulation. (b) Representative GP false-colored images of four vesicles with different GP values.
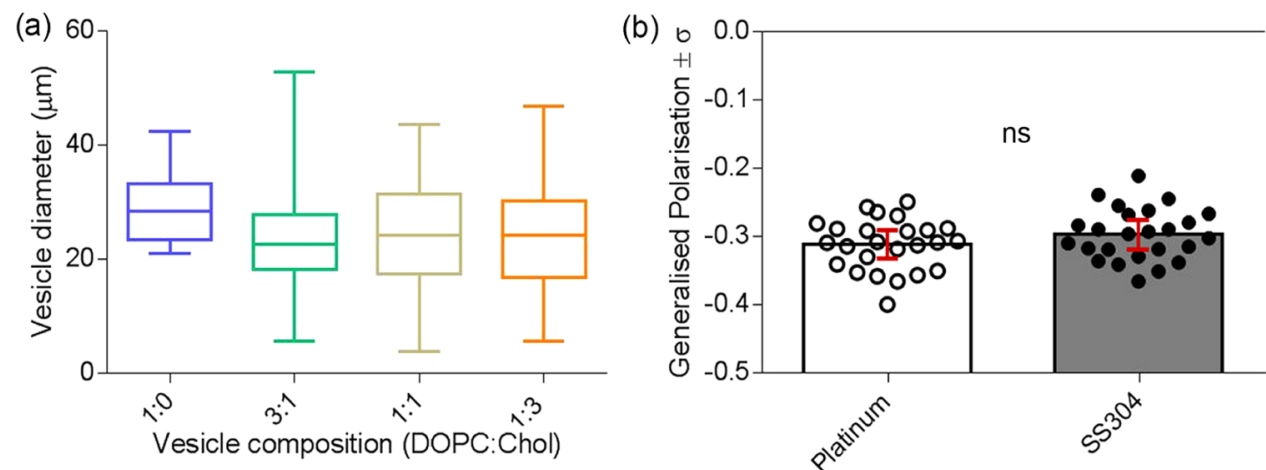

Figure 5. (a) Vesicle diameters as a function of lipid composition (b) Lipid packing of DOPC vesicles as a function of electrode material.

described in the Materials and Methods section. Vesicle formation was observed across most of the electrode surface, even before the application of the electric field (Figure 2a). Upon switching on the signal generator, the swelling was accelerated, and the vesicles reached a moderate size (see video in the Supporting Information). Swelling and detachment of DOPC vesicles from an electrode were also observed (Figure $2 \mathrm{~b}, \mathrm{c})$.

Following the visualization, vesicles were produced inside the device depicted in Figure 2a using the electroformation protocol described in the Materials and Methods section. The electrode material did not produce significant differences in the size distributions of the vesicles (Figure 3) which were within the ranges reported in the literature $(10-30 \mu \mathrm{m}$ for DOPC vesicles). ${ }^{27,31,32}$

The yield of a single electroformation chamber was estimated by suspending $100 \mu \mathrm{L}$ of a GUV-rich solution in phosphatebuffered saline (PBS) and counting the number of vesicles at the bottom of a visualization plate after sedimentation. The vesicles were counted using a purpose-built algorithm for both 


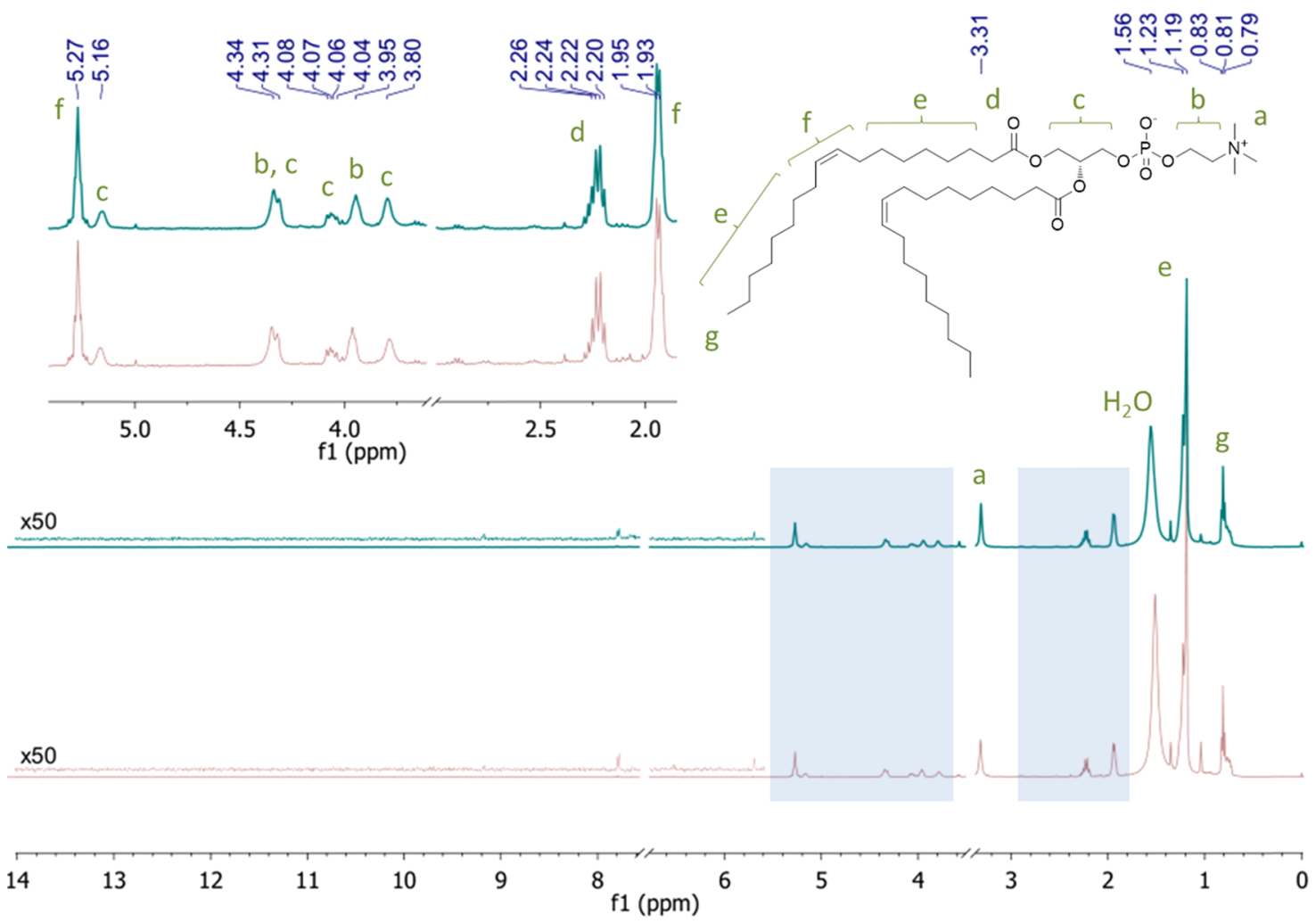

Figure 6. ${ }^{1} \mathrm{H}$ NMR spectra of lipids extracted from electroformed GUVs (top trace) and large multilamellar vesicles (LMVs) (bottom trace). The residual solvent peaks of chloroform $(7.26 \mathrm{ppm})$ and methanol $(3.49 \mathrm{ppm})$ were removed for clarity. The inset shows a magnified view of the olefinic and head group protons.

electrode materials. On average, electroformation of the stainless steel electrode yielded 2256 vesicles (22 560 vesicles $/ \mathrm{mL}$ ) against the 3114 (31 140 vesicles $/ \mathrm{mL}$ ) yielded by the platinum wire electrodes. The difference, however, was not statistically significant.

It should be noted, however, that a comparison between GUV studies is problematic because of the differences in experimental parameters, such as osmotic pressure, electrode size and separation, electroformation solution, lipid species, electric field strength, imaging plane, and vesicle manipulation technique.

Lipid Order and Size of Vesicles Composed of Binary Lipid Mixtures. To test the parallelization potential, vesicles with different compositions were produced simultaneously by varying the DOPC/cholesterol (DOPC/Chol) molar ratio in the multichamber device (Figure 4).

The results depicted in Figure 5 were obtained by performing four concurrent electroformation experiments, coating the wires in each chamber with a different lipid composition. Figure 5a displays the average GPs and the standard deviations of the vesicles for each initial lipid composition. The packing of the membrane increases with increasing cholesterol volume fraction, as cholesterol intercalates between the DOPC lipids. ${ }^{33-35}$ The electroformation cycle lasted $2.5 \mathrm{~h}$, required limited setup time, and was performed using four pairs of new injection needles (SS304), at a total electrode cost of approximately $£ 2.00$. The equivalent cost of Pt wire electrodes would have been approximately $£ 584$ and would have required electrode cleaning and straightening.

The consistency in vesicle diameter was determined as a function of lipid composition. The vesicle size was not affected significantly by the molar fraction of cholesterol compared with the 1:0 DOPC/Chol composition (Figure 6a). The electrode material did not have an effect on the lipid packing of GUV electrodes (Figure 6b). A higher osmolarity of the extravesicular environment yields vesicles with a higher membrane order and thus a higher degree of lipid packing. ${ }^{36}$

Leaching of Metals from the Electrodes. Leaching of transition metals from the electrodes can be a concern in the electroformation of GUVs. Metal ions can bind to membranes $^{37}$ and alter their bilayer structure, ${ }^{38}$ phase behavior, ${ }^{39}$ and stability against vesicle fusion. ${ }^{40}$ Unwanted chemical reactions can also be catalyzed by trace metals: chromium, iron, and nickel can initiate and, in some cases, propagate lipid peroxidation. $^{41}$

The possibility of leaching of metals from stainless steel electrodes under electroformation conditions was assessed by comparing the concentrations of their constituents $(\mathrm{Fe}, \mathrm{Cr}, \mathrm{Ni}$, and $\mathrm{Mn}$ ) in GUV samples (Table 2) prepared using stainless steel and platinum electrodes. No increase in metal concentration compared to that of platinum electrodes was

Table 2. Concentrations of Alloy Constituents Inside the Electroformation Chamber ${ }^{a}$

$\begin{array}{lccc}\text { element } & \begin{array}{c}\text { \% fraction } \\ \text { in SS304 }\end{array} & \begin{array}{c}\text { concentration with Pt } \\ \text { electrodes (ppm) }\end{array} & \begin{array}{c}\text { concentration with } \\ \text { SS304 electrodes } \\ \text { (ppm) }\end{array} \\ \text { iron } & 65-71 & <0.1 & <0.1 \\ \text { chromium } & 18-20 & <0.1 & <0.1 \\ \text { nickel } & 8-12 & <0.1 & <0.1 \\ \text { manganese } & 2 & <0.1 & <0.1\end{array}$

${ }^{a}$ The concentrations of both platinum and stainless steel electrodes are shown. 
detected by ICP-OES. While no evidence of ion leaching was detected when the electroformation was carried out in $200 \mathrm{mM}$ sucrose, this may not be the case in conductive electroformation solutions. On the contrary, platinum electrodes are routinely used to electroform vesicles in solutions of physiological ionic strength. A similar argument holds true for temperature, as the electroformation of vesicles with higher transition temperatures requires a heated chamber.

Lipid Oxidation and Hydrolysis. The products of lipid oxidation and hydrolysis, which can be generated at the electrode during the electroformation process, ${ }^{42}$ are also known to affect the physical properties of phospholipid membranes. Even small amounts of the degradation products can cause structural and dynamic changes, such as the formation of lipid rafts $^{42}$ or changes in permeability ${ }^{43}$ and mechanical stability. ${ }^{44}$ ${ }^{1} \mathrm{H}$ NMR and ${ }^{31} \mathrm{P}$ NMR spectroscopies are among the fastest and most informative analytical methods that have been used to characterize the chemical structure of phospholipids extracted from biological membranes and their degradation products. $^{45-48}$ The structural integrity of electroformed GUVs was assessed by comparing the NMR spectra of the combined lipid extracts from nine electroformed GUV samples with a control obtained from LMVs prepared by lipid hydration. The ${ }^{1} \mathrm{H}$ NMR spectra of lipids extracted from electroformed GUVs and LMVs are compared in Figure 6. The spectra were identical and consistent with pure DOPC. No degradation products were detected within a limit of detection of $1 \mathrm{~mol} \%$. Although trace impurities can be seen in the electroformed sample, the same impurities are found in the control. Their concentration remains constant when the concentration of DOPC in the control sample is increased from 0.34 to $3.4 \mathrm{mM}$, suggesting that they could be due to trace contamination rather than lipid degradation.

The absence of hydrolysis products was confirmed by the ${ }^{31} \mathrm{P}$ NMR spectrum, which shows only one signal in both samples. All isomers of lyso-PC would resonate downfield, where no signal was detected.

Unilamellarity of Electroformed Vesicles. Vesicle unilamellarity was tested using a fluorescence quenching assay on the basis of the measurement of the ratio of inner to outer layers of fluorescently labeled GUVs. ${ }^{49,50}$ DOPC vesicles symmetrically labeled with the fluorescent lipid, $N$-(7-nitrobenz-2-oxa-1,3-diazol-4-yl)-1,2-dihexadecanoyl-sn-glycero-3phosphoethanolamine, triethylammonium salt (NBD-PE), were electroformed, and a time course of their fluorescence was measured in a plate reader. Sodium dithionite, a membraneimpermeable quencher, was added to the sample to quench the fluorophores on the outer layer of the vesicles. The vesicles were then lysed by addition of Triton X-100, to expose all lipids to the quencher. The ratio of the emission drop caused by the addition of detergent $\left(I_{\text {interior }}\right)$ to the total emission drop $\left(I_{\text {total }}=\right.$ $\left.I_{\text {interior }}+I_{\text {exterior }}\right)$ is used as a measure of the fraction of leaflets that are not exposed to the solvent (unilamellarity index) (Figure 7 ). We measured a ratio of $51 \pm 2.8 \%$, which is consistent with unilamellarity. The measurements for all three samples are provided in the Supporting Information.

\section{CONCLUSIONS}

In summary, in this article, giant vesicle electroformation using an alternative electrode material was proposed and validated. We demonstrate that electroswelling of unilamellar vesicles occurs efficiently on stainless steel electrodes without any significant difference in vesicle size distribution, lipid

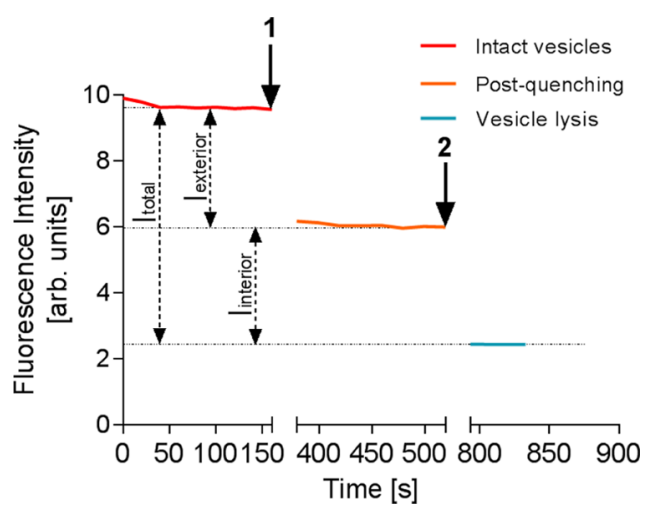

Figure 7. Fluorescence intensity measurements of DOPC/NBD-PE vesicles. The fluorescence of intact vesicles is represented from time $=$ 0 to arrow 1 . At arrow 1 , the quencher is added to the suspension, and the intensity is recorded until a stable value is reached. The emission drop is a measure of the number of fluorophores in outer leaflets. At arrow 2, Triton X-100 is added to lyse the vesicles, and fluorescence is recorded until a new stable value is reached. The corresponding emission drop is a measure of the number of fluorophores in inner leaflets. After each addition, the plate was removed from the plate reader and shaken for several minutes (the time axis is correspondingly cut for clarity).

degradation, or leaching of metals in the electroformation chamber from those of platinum electrodes. By using readily available stainless steel electrodes, we show that rapid, low-cost, and scalable electroformation can be achieved. We believe this platform will find application in biophysical investigations of the membrane, particularly in studies where multiplexing is crucial, such as large-scale screening of bioactive compounds.

\section{MATERIALS AND METHODS}

Electroformation Chamber. The electroformation chamber (Figure 8a) is composed of two parts: the electroformation base and the chamber lid. The electroformation base $(150 \mathrm{~mm}$ $\times 40 \mathrm{~mm} \times 50 \mathrm{~mm}$ ) was designed to accommodate five equidistant electroformation chambers with a length of $50 \mathrm{~mm}$ and a diameter of $10 \mathrm{~mm}$. The extremities of the chambers were tapered to increase vesicle concentration and facilitate the collection of the produced GUVs. The electroformation lid $(150 \mathrm{~mm} \times 40 \mathrm{~mm} \times 5 \mathrm{~mm})$ consists of five pairs of through holes $(\varnothing 1 \mathrm{~mm})$, each aligned with an electroformation chamber, that are used to host the electrodes. On the side of the lid, $3 \mathrm{~mm}$ brass screws were positioned in correspondence to each electroformation chamber, to hold the electrodes in place once fully screwed. A copper bar $(165 \mathrm{~mm} \times 10 \mathrm{~mm})$ ensures an electrical connection between corresponding electrodes. The chamber base and the lid were held together with four $4 \mathrm{~mm}$ screws at each corner of the device. The cost of the electroformation device with five electroformation chambers was calculated to be $\sim £ 14.00$. The full cost breakdown is provided in the Supporting Information.

A similar device (Figure $8 \mathrm{~b}$ ) was designed with the aim of visualizing the vesicle electroswelling process. The device was designed to be dimensionally compatible with a microscope stage and the working distance of a $40 \times$ objective. In this device, the electroformation electrodes were placed horizontally to allow imaging of the entire electrode surface area. A $170 \mu \mathrm{m}$ thick glass slide was coupled with the bottom of the chamber with silicone gel. The electrodes were connected using two 

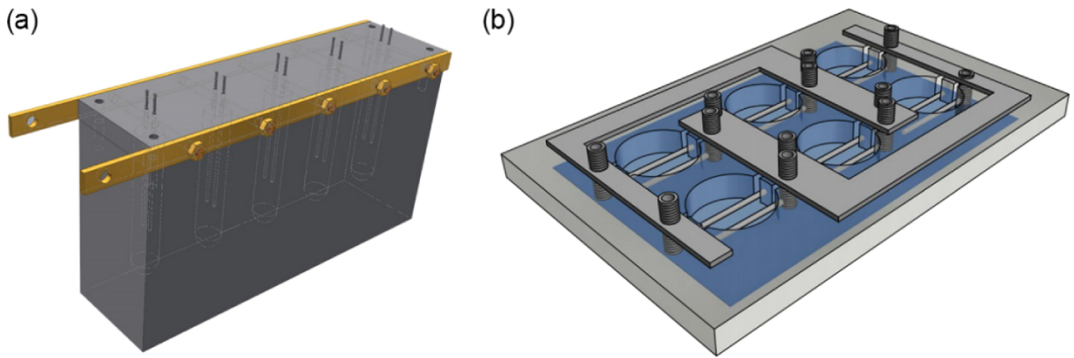

Figure 8. Vesicle electroformation devices for high-yield production and formation visualization. (a) Scalable device with five electroformation chambers and needles held by brass screws. (b) Monitoring device with six chambers, brass screws, and stainless steel sheets for electrical connection between common electrodes.

sheets of stainless steel, to provide a common electrical connection.

The electroformation chamber was cut from a solid piece of polyoxymethylene purchased from RS Components, U.K., brass screws were also supplied by RS Components, and $0.8 \mathrm{~mm} 21 \mathrm{G}$ $50 \mathrm{~mm}$ needles were purchased from Becton, Dickinson and Company. DOPC was sourced from Avanti Polar Lipids, Inc.. Cholesterol, sucrose, 2-[4-(2-hydroxyethyl)piperazin-1-yl]ethanesulfonic acid (HEPES), and bovine serum albumin (BSA) were purchased from Sigma-Aldrich. NBD-PE was purchased from ThermoFisher, U.K. All reagents were used as received without further purification. The electrodes were connected to an Agilent 33220A signal generator set to highimpedance load. GUVs were imaged in a $\mu$-Slide eight-well multiwell plate purchased from ibidi $\mathrm{GmbH}$, Germany. Ultrapure water (MilliQ) from a Millipore filtration system (resistivity, $>18.2 \mathrm{M} \Omega \mathrm{cm}$ ) was used throughout all experiments.

Electroformation Protocol. The electroformation protocol consisted in preparing solutions of DOPC $(1 \mathrm{mg} / \mathrm{mL}$ in chloroform) and cholesterol (10 $\mathrm{mg} / \mathrm{mL}$ in chloroform) at varying molar ratios $(1: 0-1: 3)$. The electrodes were rinsed in toluene and wiped using Kimwipes (Kimberly-Clark Professional) to remove any traces of silicon lubricant. The desired solution was then pipetted vertically onto the electrodes $(50 \mu \mathrm{L}$ per electrode pair) using a glass syringe, carefully coating the entire surface. After a drying phase of approximately $1 \mathrm{~h}$, the electroformation chamber was filled with a sucrose solution ( $\sim 3 \mathrm{~mL}, 200 \mathrm{mM}$ sucrose in deionized (DI) water at room temperature), the electrodes were submerged, and the signal generator was connected via the brass screws on the sides of the chamber.

A $5 \mathrm{Vpp}, 10 \mathrm{~Hz}$ sinusoidal excitation was applied for $2 \mathrm{~h}$ to induce repetitive stress on the hydrated lipid bilayer, leading to vesicle swelling. The effective voltage on the electroformation chamber was recorded using a high-voltage probe and oscilloscope as $4.87 \mathrm{Vpp}$, indicating a $0.13 \mathrm{~V}$ voltage difference due to an unmatched load of the signal generator. Subsequently, the frequency of the excitation was lowered to $5 \mathrm{~Hz}$ for $30 \mathrm{~min}$ to facilitate vesicle detachment. The electroformation parameters used in the protocol are summarized in Table 3. At the end of the electroformation process, the lid of the chamber was removed and the vesicles were slowly pipetted into a visualization well using a 100-1000 $\mu \mathrm{L}$ pipette tip. To minimize the shear stress at the orifice, the end of the pipette tip was cut by $2 \mathrm{~mm}$ using a pair of scissors. The same protocol was used for both SS304 and platinum electrodes.
Table 3. Electroformation Signal Generator Parameters for GUV Formation

\begin{tabular}{cccc} 
& \multicolumn{2}{c}{ parameters } & \\
\cline { 2 - 3 } electroformation phase & voltage $(\mathrm{V})$ & frequency $(\mathrm{Hz})$ & time (min) \\
I & 5 & 10 & 120 \\
II & 5 & 5 & 30
\end{tabular}

GUV Detection and Sizing. The GUVs were imaged by phase-contrast microscopy using a Nikon ECLIPSE Ti inverted microscope (Nikon Corporation, Japan), and the images were then processed using a purpose-built MATLAB routine. The algorithm detects the GUVs on the basis of the imfindcircles built-in MATLAB function, calculates their diameter, and plots a vesicle-size histogram for all of the vesicles detected.

To quantify the concentration of DOPC GUVs, a finite volume $(100 \mu \mathrm{L})$ of the electroformation solution was placed in an 8-well imaging dish (ibidi, U.K.) with $400 \mu \mathrm{L}$ of PBS. The dish was coated with BSA by letting a $1 \mathrm{mg} / \mathrm{mL}$ BSA solution in DI water stand for $2 \mathrm{~h}$. The BSA solution was then rinsed gently with DI water. After allowing the GUVs to sediment for an additional $2 \mathrm{~h}$, the glass surface of the dish was imaged to cover the full area of the dish. A bounding grid that consisted of $39 \times 45$ tiles with $10 \%$ overlap was used for stitching.

Lipid Order Measurements. The lateral organization of the membrane lipids, also referred to as lipid packing, was quantified using a microscope-based spectral imaging technique. ${ }^{3,51}$ For this purpose, an aliquot $(100 \mu \mathrm{L})$ of GUV-rich solution was placed in an imaging well. The vesicles were then fluorescently labeled with a final concentration of $400 \mathrm{nM}$ cLaurdan, an environment-sensitive molecular probe. The solution was then diluted with $100 \mu \mathrm{L}$ of PBS. In the case of the experiment relating lipid packing to electrode material, 400 $\mu \mathrm{L}$ of PBS was added to the solution.

The emission spectrum of c-Laurdan shifts as a function of the dipolar water relaxation and thus the level of hydration within its surrounding microenvironment, indicating lipid packing in membranes. $\mathrm{GP}^{52}$ was employed as a relative measure of lipid packing on a scale of -1 to 1 , where -1 represents the least-packed membrane and 1 represents the most-packed membrane. Equation 1 describes the GP relative to the intensities at two specific wavelengths: $440\left(I_{440}\right)$ and $490 \mathrm{~nm}\left(I_{490}\right)$.

$$
\mathrm{GP}=\frac{I_{440}-I_{490}}{I_{440}+I_{490}}
$$

Spectral imaging was performed using a Zeiss LSM780 confocal microscope equipped with a 32-channel GaAsP detector array. 
The vesicles were excited at $405 \mathrm{~nm}$, and the spectral intensity of the signal was recorded in the 415-691 nm range.

ICP-OES Experiments. The concentration of metal ions in the electroformed samples was measured by ICP-OES with a Varian Vista-MPX spectrometer, for both electrode materials.

Lipid Extraction and NMR Experiments. The samples for NMR analysis were prepared from nine simultaneous electroformation experiments. The content of each electroformation chamber $(3 \mathrm{~mL})$ was diluted with an equal volume of PBS in a $50 \mathrm{~mL}$ Falcon tube, which was then left to stand overnight at room temperature. The GUV-enriched layers (500 $\mu \mathrm{L}$ each) were then withdrawn from the bottom of each Falcon tube, combined, and freeze-dried. The lipids were then extracted from the dried GUV samples by the Bligh and Dyer method. ${ }^{53}$ Briefly, the sample was dispersed in water $(0.8$ $\mathrm{mL})$, and then methanol $(2 \mathrm{~mL})$ and chloroform $(1 \mathrm{~mL})$ were added with mixing after each addition. The sample was vortexed at $2500 \mathrm{rpm}$ for $15 \mathrm{~s}$ and allowed to stand for $30 \mathrm{~min}$. Chloroform $(1 \mathrm{~mL})$ and water $(1 \mathrm{~mL})$ were added, and the sample was centrifuged at $1000 \mathrm{~g}$ for $5 \mathrm{~min}$ to achieve a complete phase separation. The bottom organic layer was withdrawn using a glass syringe, dried under a nitrogen stream, and redissolved in $\mathrm{CDCl}_{3}(600 \mu \mathrm{L})$ for analysis.

LMV control samples with different lipid concentrations were prepared using the lipid hydration method. A solution of DOPC in chloroform was added to a glass vial and dried under a nitrogen stream. The lipid film was then hydrated by adding the same sucrose solution used for electroformation and vortexed for $2 \mathrm{~min}$ at $2500 \mathrm{rpm}$. After adding an equal volume of PBS, the sample was left to stand at room temperature for 18 h. The lipids were then extracted with the same protocol used for the electroformed sample.

${ }^{1} \mathrm{H}$ NMR spectra were acquired on a Bruker Ascend 400 spectrometer (at $400 \mathrm{MHz}$ for ${ }^{1} \mathrm{H}$ and at $162 \mathrm{MHz}$ for ${ }^{31} \mathrm{P}$ ) with $30^{\circ}$ pulses (Bruker $\mathrm{zg} 30$ sequence) and a $3 \mathrm{~s}$ relaxation delay on a spectral width of $8000 \mathrm{~Hz}$, whereas ${ }^{31} \mathrm{P}$ NMR spectra were acquired with proton decoupling and a $3 \mathrm{~s}$ relaxation delay on a spectral width of $64103 \mathrm{~Hz}$. The spectra were apodized by multiplication with an exponential decay equivalent to $0.5 \mathrm{~Hz}$ line broadening and a Gaussian function equivalent to $1 \mathrm{~Hz}$ line broadening. ${ }^{1} \mathrm{H}$ NMR spectra were referenced to residual nondeuterated chloroform, whereas ${ }^{31} \mathrm{P}$ NMR spectra were externally referenced to triphenylphosphine oxide.

Vesicle Unilamellarity. Three independent suspensions of fluorescently labeled vesicles were prepared using the electroformation protocol outlined previously, by depositing a mixture of DOPC and NBD-PE (1 mol \%) in chloroform on the electrodes. The GUV suspension $(2.7 \mathrm{~mL})$ was then mixed with $300 \mu \mathrm{L}$ of a HEPES buffer at $\mathrm{pH} 7$ (0.1 M HEPES and 1 $\mathrm{M} \mathrm{NaCl}$ ) to obtain final concentrations of $10 \mathrm{mM} \mathrm{HEPES}$ and $100 \mathrm{mM} \mathrm{NaCl}$. Three wells of a 96-well plate were filled with $200 \mu \mathrm{L}$ of the buffered GUV suspension. Stable values of fluorescence intensity were measured at $520 \mathrm{~nm}$ on a FLUOstar Omega plate reader (BMG Labtech) before and after adding 4 $\mu \mathrm{L}$ of a freshly prepared solution of $1 \mathrm{M} \mathrm{Na}_{2} \mathrm{~S}_{2} \mathrm{O}_{4}$ in $1 \mathrm{M}$ Tris buffer at $\mathrm{pH} 10$, with excitation at $485 \mathrm{~nm}$. Subsequently, $20 \mu \mathrm{L}$ of a $10 \% \mathrm{v} / \mathrm{v}$ solution of Triton X-100 was added to the solution to cause vesicle lysis and expose all lipid structures to the quencher. A final stable value of fluorescence was recorded after the addition of the detergent. The unilamellarity index, defined as the fraction of leaflets that are not exposed to the solvent, was calculated as the ratio of the emission drop caused by the addition of detergent $\left(I_{\text {interior }}\right)$ to the total emission drop $\left(I_{\text {total }}=I_{\text {interior }}+I_{\text {exterior }}\right)$.

Statistical Analyses. Statistical testing was performed using one-way analysis of variance for multiple comparison analyses, whereas a Student's $t$-test was employed for direct comparison between two data sets. All data were expressed in terms of mean \pm standard deviation, and the number of independent replicates was expressed in the figure captions. The following conventions for statistical significance are used throughout the paper: ns, $p>0.05$; $, p \leq 0.05, * *, p \leq 0.01$; ***, $p \leq 0.001$; $* * * *, p \leq 0.0001$.

\section{ASSOCIATED CONTENT}

S Supporting Information

The Supporting Information is available free of charge on the ACS Publications website at DOI: 10.1021/acsomega.6b00395.

Cost breakdown of the electroformation chamber, limit of detection in NMR experiments, and unilamellarity experimental data; photograph of electroformation chamber (PDF)

Video of the formation of vesicles on an SS304 electrode upon the application of an external electric field at $t=3 \mathrm{~s}$ $($ scale bar $=20 \mu \mathrm{m})($ AVI $)$

\section{AUTHOR INFORMATION}

\section{Corresponding Author}

*E-mail: eleanor.stride@eng.ox.ac.uk. Phone: +44 (0)1865 617747.

ORCID

Jorge Bernardino de la Serna: 0000-0002-1396-3338

Eleanor Stride: 0000-0003-3371-5929

\section{Author Contributions}

The article was written through contributions of all authors. All authors have given approval to the final version of the article.

\section{Funding}

This work was supported by EPSRC Grant EP/I021795/1. E. Sezgin and C. Eggeling thank Wolfson Foundation, the Medical Research Council (MRC, grant number MC_UU_12010/unit programmes G0902418 and MC_UU_12025), MRC/BBSRC/ ESPRC (grant number $\mathrm{MR} / \mathrm{K} 01577 \overline{\mathrm{X}} / 1$ ), and the Wellcome Trust (grant ref 104924/14/Z/14). E. Sezgin was supported by EMBO Long-Term and Marie Curie Intra-European Fellowships (MEMBRANE DYNAMICS). J. Bernardino de la Serna acknowledges support from the Marie Curie Career Integration Grant.

Notes

The authors declare no competing financial interest.

Raw data are available through the University of Oxford ORA data repository doi: 10.5287/bodleian:9ed9ON5wZ.

\section{ACKNOWLEDGMENTS}

The authors extend their gratitude to James Fisk and David Salisbury for the fabrication of the electroformation chambers, Graham Brown for helpful discussions on microscopic techniques, and Fabio Bianchi for the helpful technical discussions.

\section{ABBREVIATIONS}

GUV, giant unilamellar vesicle; LMV, large multilamellar vesicle; E, electric field; DOPC, 1,2-dioleoyl-sn-glycero-3phosphatidylcholine; DSPC, 1,2-distearoyl-sn-glycero-3-phos- 
phocholine; DPPC, 1,2-dipalmitoyl-sn-glycero-3-phosphocholine; DMPC, 1,2-dimyristoyl-sn-glycero-3-phosphocholine; ITO, indium tin oxide; ${ }^{1} \mathrm{H} \mathrm{NMR}$, proton nuclear magnetic resonance; ICP-OES, inductively coupled plasma optical emission spectrometry; PBS, phosphate-buffered saline; Vpp, peak-to-peak voltage

\section{REFERENCES}

(1) Lingwood, D.; Simons, K. Lipid Rafts as a Membrane-Organizing Principle. Science 2010, 327, 46-50.

(2) Bagatolli, L. A.; Gratton, E. A Correlation between Lipid Domain Shape and Binary Phospholipid Mixture Composition in Free Standing Bilayers: A Two-Photon Fluorescence Microscopy Study. Biophys. J. 2000, 79, 434-447.

(3) Dietrich, C.; Bagatolli, L. A.; Volovyk, Z. N.; Thompson, N. L.; Levi, M.; Jacobson, K.; Gratton, E. Lipid Rafts Reconstituted in Model Membranes. Biophys. J. 2001, 80, 1417-1428.

(4) Korlach, J.; Schwille, P.; Webb, W. W.; Feigenson, G. W. Characterization of Lipid Bilayer Phases by Confocal Microscopy and Fluorescence Correlation Spectroscopy. Proc. Natl. Acad. Sci. U.S.A. 1999, 96, 8461-8466.

(5) Feigenson, G. W.; Buboltz, J. T. Ternary Phase Diagram of Dipalmitoyl-PC/Dilauroyl-PC/Cholesterol: Nanoscopic Domain Formation Driven by Cholesterol. Biophys. J. 2001, 80, 2775-2788.

(6) Veatch, S. L.; Keller, S. L. Separation of Liquid Phases in Giant Vesicles of Ternary Mixtures of Phospholipids and Cholesterol. Biophys. J. 2003, 85, 3074-3083.

(7) Sun, T.-L.; Sun, Y.; Lee, C.-C.; Huang, H. W. Membrane Permeability of Hydrocarbon-Cross-Linked Peptides. Biophys. J. 2013, 104, 1923-1932.

(8) Nishimura, K.; Matsuura, T.; Sunami, T.; Fujii, S.; Nishimura, K.; Suzuki, H.; Yomo, T. Identification of Giant Unilamellar Vesicles with Permeability to Small Charged Molecules. RSC Adv. 2014, 4, 35224.

(9) Staykova, M.; Lipowsky, R.; Dimova, R. Membrane Flow Patterns in Multicomponent Giant Vesicles Induced by Alternating Electric Fields. Soft Matter 2008, 4, 2168.

(10) Fenz, S. F.; Sengupta, K. Giant Vesicles as Cell Models. Integr. Biol. 2012, 4, 982.

(11) Nappini, S.; Al Kayal, T.; Berti, D.; Nordèn, B.; Baglioni, P. Magnetically Triggered Release From Giant Unilamellar Vesicles: Visualization By Means Of Confocal Microscopy. J. Phys. Chem. Lett. 2011, 2, 713-718.

(12) Dubavik, A.; Sezgin, E.; Lesnyak, V.; Gaponik, N.; Schwille, P.; Eychmüller, A. Penetration of Amphiphilic Quantum Dots through Model and Cellular Plasma Membranes. ACS Nano 2012, 6, 21502156.

(13) Bernardino de la Serna, J.; Perez-Gil, J.; Simonsen, A. C.; Bagatolli, L. A. Cholesterol Rules. J. Biol. Chem. 2004, 279, 4071540722 .

(14) Bernardino de la Serna, J.; Hansen, S.; Berzina, Z.; Simonsen, A. C.; Hannibal-Bach, H. K.; Knudsen, J.; Ejsing, C. S.; Bagatolli, L. A. Compositional and Structural Characterization of Monolayers and Bilayers Composed of Native Pulmonary Surfactant from Wild Type Mice. Biochim. Biophys. Acta, Biomembr. 2013, 1828, 2450-2459.

(15) Heinrich, M. C.; Capraro, B. R.; Tian, A.; Isas, J. M.; Langen, R.; Baumgart, T. Quantifying Membrane Curvature Generation of Drosophila Amphiphysin N-BAR Domains. J. Phys. Chem. Lett. 2010, 1, 3401-3406.

(16) López-Montero, I.; Rodríguez-García, R.; Monroy, F. Artificial Spectrin Shells Reconstituted on Giant Vesicles. J. Phys. Chem. Lett. 2012, 3, 1583-1588.

(17) Caschera, F.; Bernardino de la Serna, J.; Löffler, P. M. G.; Rasmussen, T. E.; Hanczyc, M. M.; Bagatolli, L. A.; Monnard, P.-A. Stable Vesicles Composed of Monocarboxylic or Dicarboxylic Fatty Acids and Trimethylammonium Amphiphiles. Langmuir 2011, 27, 14078.

(18) Walde, P.; Cosentino, K.; Engel, H.; Stano, P. Giant Vesicles: Preparations and Applications. ChemBioChem 2010, 11, 848-865.
(19) Méléard, P.; Bagatolli, L. A.; Pott, T. Giant Unilamellar Vesicle Electroformation: From Lipid Mixtures to Native Membranes under Physiological Conditions. Methods Enzymol. 2009, 465, 161-176.

(20) Dimova, R.; Aranda, S.; Bezlyepkina, N.; Nikolov, V.; Riske, K. A.; Lipowsky, R. A Practical Guide to Giant Vesicles. Probing the Membrane Nanoregime via Optical Microscopy. J. Phys.: Condens. Matter 2006, 18, S1151-76.

(21) Reeves, J. P.; Dowben, R. M. Formation and Properties of ThinWalled Phospholipid Vesicles. J. Cell. Physiol. 1969, 73, 49-60.

(22) Angelova, M. I.; Dimitrov, D. S. Liposome Electroformation. Faraday Discuss. Chem. Soc. 1986, 81, 303.

(23) Pautot, S.; Frisken, B. J.; Weitz, D. A. Production of Unilamellar Vesicles Using an Inverted Emulsion. Langmuir 2003, 19, 2870-2879.

(24) Yamada, A.; Le Berre, M.; Yoshikawa, K.; Baigl, D. Spontaneous Generation of Giant Liposomes from an Oil/water Interface. ChemBioChem 2007, 8, 2215-2218.

(25) Stachowiak, J. C.; Richmond, D. L.; Li, T. H.; Liu, A. P.; Parekh, S. H.; Fletcher, D. A. Unilamellar Vesicle Formation and Encapsulation by Microfluidic Jetting. Proc. Natl. Acad. Sci. U.S.A. 2008, 105, 4697-4702.

(26) Arriaga, L. R.; Datta, S. S.; Kim, S.-H.; Amstad, E.; Kodger, T. E.; Monroy, F.; Weitz, D. A. Ultrathin Shell Double Emulsion Templated Giant Unilamellar Lipid Vesicles with Controlled Microdomain Formation. Small 2014, 10, 950-956.

(27) Bi, H.; Yang, B.; Wang, L.; Cao, W.; Han, X. Electroformation of Giant Unilamellar Vesicles Using Interdigitated ITO Electrodes. J. Mater. Chem. A 2013, 1, 7125.

(28) Aimon, S.; Manzi, J.; Schmidt, D.; Poveda Larrosa, J. A.; Bassereau, P.; Toombes, G. E. S. Functional Reconstitution of a Voltage-Gated Potassium Channel in Giant Unilamellar Vesicles. PLoS One 2011, 6, No. e25529.

(29) Fidorra, M.; Duelund, L.; Leidy, C.; Simonsen, A. C.; Bagatolli, L. A. Absence of Fluid-Ordered/Fluid-Disordered Phase Coexistence in Ceramide/POPC Mixtures Containing Cholesterol. Biophys. J. 2006, 90, 4437-4451.

(30) Montes, L.-R.; Alonso, A.; Goñi, F. M.; Bagatolli, L. A. Giant Unilamellar Vesicles Electroformed from Native Membranes and Organic Lipid Mixtures under Physiological Conditions. Biophys. J. 2007, 93, 3548-3554.

(31) Lecuyer, S.; Ristenpart, W. D.; Vincent, O.; Stone, H. A. Electrohydrodynamic Size Stratification and Flow Separation of Giant Vesicles. Appl. Phys. Lett. 2008, 92, No. 104105.

(32) Herold, C.; Chwastek, G.; Schwille, P.; Petrov, E. P. Efficient Electroformation of Supergiant Unilamellar Vesicles Containing Cationic Lipids on ITO-Coated Electrodes. Langmuir 2012, 28, $5518-5521$.

(33) Sezgin, E.; Sadowski, T.; Simons, K. Measuring Lipid Packing of Model and Cellular Membranes with Environment Sensitive Probes. Langmuir 2014, 30, 8160-8166.

(34) Silvius, J. R. Role of Cholesterol in Lipid Raft Formation: Lessons from Lipid Model Systems. Biochim. Biophys. Acta, Biomembr. 2003, 1610, 174-183.

(35) Veatch, S. L.; Keller, S. L. Organization in Lipid Membranes Containing Cholesterol. Phys. Rev. Lett. 2002, 89, No. 268101.

(36) Söderlund, T.; Alakoskela, J.-M. I.; Pakkanen, A. L.; Kinnunen, P. K. J. Comparison of the Effects of Surface Tension and Osmotic Pressure on the Interfacial Hydration of a Fluid Phospholipid Bilayer. Biophys. J. 2003, 85, 2333-2341.

(37) Lis, L. J.; Lis, W. T.; Parsegian, V. A.; Rand, R. P. Adsorption of Divalent Cations to a Variety of Phosphatidylcholine Bilayers. Biochemistry 1981, 20, 1771-1777.

(38) Suwalsky, M.; Castro, R.; Villena, F.; Sotomayor, C. P. Cr(III) Exerts Stronger Structural Effects than $\mathrm{Cr}(\mathrm{VI})$ on the Human Erythrocyte Membrane and Molecular Models. J. Inorg. Biochem. 2008, 102, 842-849.

(39) Binder, H.; Zschörnig, O. The Effect of Metal Cations on the Phase Behavior and Hydration Characteristics of Phospholipid Membranes. Chem. Phys. Lipids 2002, 115, 39-61. 
(40) Ohki, S.; Düzgünes, N. Divalent Cation-Induced Interaction of Phospholipid Vesicle and Monolayer Membranes. Biochim. Biophys. Acta, Biomembr. 1979, 552, 438-449.

(41) Stohs, S. J.; Bagchi, D. Oxidative Mechanisms in the Toxicity of Metal Ions. Free Radicals Biol. Med. 1995, 18, 321-336.

(42) Ayuyan, A. G.; Cohen, F. S. Lipid Peroxides Promote Large Rafts: Effects of Excitation of Probes in Fluorescence Microscopy and Electrochemical Reactions during Vesicle Formation. Biophys. J. 2006, 91, 2172-2183.

(43) Mills, J. K.; Needham, D. Lysolipid Incorporation in Dipalmitoylphosphatidylcholine Bilayer Membranes Enhances the Ion Permeability and Drug Release Rates at the Membrane Phase Transition. Biochim. Biophys. Acta, Biomembr. 2005, 1716, 77-96.

(44) Fuller, N.; Rand, R. P. The Influence of Lysolipids on the Spontaneous Curvature and Bending Elasticity of Phospholipid Membranes. Biophys. J. 2001, 81, 243-254.

(45) Sparling, M. L.; Zidovetzki, R.; Muller, L.; Chan, S. I. Analysis of Membrane Lipids by $500 \mathrm{MHz} 1 \mathrm{H}$ NMR. Anal. Biochem. 1989, 178, 67-76.

(46) Nieva-Echevarría, B.; Goicoechea, E.; Manzanos, M. J.; Guillén, M. D. Usefulness of $1 \mathrm{H}$ NMR in Assessing the Extent of Lipid Digestion. Food Chem. 2015, 179, 182-190.

(47) Kriat, M.; Vion-Dury, J.; Confort-Gouny, S.; Favre, R.; Viout, P.; Sciaky, M.; Sari, H.; Cozzone, P. J. Analysis of Plasma Lipids by NMR Spectroscopy: Application to Modifications Induced by Malignant Tumors. J. Lipid Res. 1993, 34, 1009-1019.

(48) MacKenzie, A.; Vyssotski, M.; Nekrasov, E. Quantitative Analysis of Dairy Phospholipids by 31P NMR. J. Am. Oil Chem. Soc. 2009, 86, 757-763.

(49) McIntyre, J. C.; Sleight, R. G. Fluorescence Assay for Phospholipid Membrane Asymmetry. Biochemistry 1991, 30, 1181911827.

(50) Gruber, H. J.; Schindler, H. External Surface and Lamellarity of Lipid Vesicles: A Practice-Oriented Set of Assay Methods. Biochim. Biophys. Acta, Biomembr. 1994, 1189, 212-224.

(51) Sezgin, E.; Waithe, D.; Bernardino de la Serna, J.; Eggeling, C. Spectral Imaging to Measure Heterogeneity in Membrane Lipid Packing. ChemPhysChem 2015, 16, 1387-1394.

(52) Parasassi, T.; De Stasio, G.; Ravagnan, G.; Rusch, R. M.; Gratton, E. Quantitation of Lipid Phases in Phospholipid Vesicles by the Generalized Polarization of Laurdan Fluorescence. Biophys. J. 1991, 60, 179-189.

(53) Bligh, E. G.; Dyer, W. J. A Rapid Method of Total Lipid Extraction and Purification. Can. J. Biochem. Physiol. 1959, 37, 911. 